\title{
Work Transfer Analysis of Turbojet and Turbofan Engines
}

\author{
Bryce A. Roth ${ }^{*}$ \\ Georgia Institute of Technology, Atlanta, GA 30332-0150
}

\begin{abstract}
This paper explores the application of work transfer analysis to turbojet and turbofan engines. Emphasis is placed on understanding and applying the basic analysis method. The relationships between component performance and overall system work transfer are explored and demonstrated for ramjet, turbojet, and turbofan examples. The analysis results obtained via this method are quantified for a separate flow turbofan example. Finally, this paper suggests groupings of parameters of fundamental importance to turbofan engines based on the analysis results developed herein.
\end{abstract}

\section{Introduction}

$\mathrm{T}$ he conventional thought process with respect to analysis of air-breathing propulsion systems focuses heavily on the flow of air through the propulsion system. In point of fact, the ultimate aim of any propulsion system is to efficiently convert energy stored in the chemical bonds of a fuel into useful thrust work. It therefore follows that what truly matters from a thermodynamic perspective is how effectively the work potential stored in the fuel is converted into useful thrust work on the vehicle. Air is only an intermediary in this process. This is the basic idea and philosophy underlying the concept of Work Transfer Analysis.

The intent of this paper is to explore work transfer analysis with specific application to typical Brayton engine cycles. This work builds on ideas explored in Ref. 1 and tailors the basic concepts to analysis of turbofan engines of current practical interest. The development is a logical extension of the work transfer concept starting with the analysis of the simple ramjet engine. The ramjet analysis is used to illustrate basic principles and is a pedagogical stepping stone towards turbofan engines. The method is subsequently applied to turbojet and turbofan engine cycles, with practical examples and results. This ultimately leads to a framework for analysis of thermodynamic efficiency of gas turbine engines.

This framework is complementary to existing frameworks described by Lewis ${ }^{2}$ and is a useful tool in understanding theoretical transfer of useable work potential through a machine. The basic model suggested in Ref. 1 for analysis of work transfer in a propulsion system is given by:

$$
w_{\text {Thrust }}=\eta_{o} L H V=\underbrace{\frac{w_{\text {Thrust }}}{w_{K E}}}_{\eta_{P}} \underbrace{\frac{w_{K E}}{w_{\text {cycle }}}}_{\eta_{T r}} \underbrace{\frac{w_{\text {cycle }}}{w_{\text {fuel }}}}_{\eta_{C T}} \underbrace{\frac{w_{\text {fuel }}}{L H V}}_{\eta_{C A}} L H V
$$

where: $\quad \mathrm{LHV}=$ Heat content (lower heating value) of the fuel per unit mass fuel

$\mathrm{w}_{\text {fuel }}=$ Maximum cycle work theoretically available in the fuel per unit mass flow

$\mathrm{w}_{\mathrm{cycle}}=$ Net work produced by the engine cycle (gas generator available energy) per unit mass flow

$\mathrm{W}_{\mathrm{KE}}=$ Net change in kinetic energy of the propulsive flow per unit mass flow

$\mathrm{w}_{\text {Thrust }}=$ Net thrust work produced per unit mass flow

$\eta_{\mathrm{o}}=$ overall propulsion system efficiency (defined as quotient of thrust work out to heat input)

$\eta_{\mathrm{P}}=$ propulsive efficiency (defined as quotient of thrust work out to kinetic energy production).

$\eta_{\text {Tr }}=$ propulsive transfer efficiency (defined as quotient of kinetic energy production to cycle work output).

$\eta_{\mathrm{CT}}=$ cycle transfer efficiency (defined as quotient of cycle work output to fuel available energy).

$\eta_{\mathrm{CA}}=$ cycle availability efficiency (defined as quotient of available energy added to the cycle to heat input).

Thus, propulsive efficiency is a measure of how much thrust work is produced per unit kinetic energy produced in the propulsive stream(s). Transfer efficiency measures how much of the net cycle work is realized as kinetic energy in the propulsive stream. Cycle transfer efficiency measures how much of the available energy added to the cycle is realized as useful output. Cycle availability efficiency measures how much of the work potential stored in the fuel is

\footnotetext{
${ }^{*}$ Research Engineer, School of Aerospace Engineering, AIAA Member.

Copyright (C) 2004 by B.A. Roth. Published by the American Institute of Aeronautics and Astronautics, Inc. with permission.
} 
realized as available energy in the cycle, measured as a fraction of the total heating value of the fuel. This model is used herein as the basis for work transfer analysis of ramjet, turbojet, and turbofan engine cycles.

\section{Pedagogical Application: Ramjet}

The best way to illustrate the concept of work transfer analysis is to apply it to a simple and well-known propulsive cycle such as the ubiquitous ramjet engine. Various portions of Eq. (1) will be developed for the case of a ramjet engine and each examined in detail. These pieces are then assembled to yield an overall model for work transfer in a ramjet engine.

\section{A. Ramjet Cycle Transfer Efficiency}

To start, consider the ramjet engine schematic shown in Fig. 1. The station numbers are noted at bottom, and the engine is assumed to be divided into the following components: inlet, combustor, and nozzle. First, flow is ingested into the inlet where its freestream kinetic energy is converted into potential energy. The ingested flow therefore has some work potential relative to the propulsion fixed reference frame by virtue of the flight speed of the propulsion system. This work potential is equal to the kinetic energy per unit mass flow of air relative to the vehicle fixed reference frame, and is denoted by $\mathrm{w}_{1}$. The inlet is not perfect and therefore the flow emerging from the inlet has some value of work potential per unit mass flow, $\mathrm{w}_{3}$, less than $\mathrm{w}_{1}$. Let us denote the ratio of $\mathrm{w}_{3} / \mathrm{w}_{1}$ as the inlet work transfer function, $\mathrm{X}_{\text {inlet. }}$. Therefore, $\mathrm{w}_{3}$ can be expressed as the product of $\mathrm{w}_{1}$ and the inlet work transfer function. Presumably, $\mathrm{w}_{1}$ is only a function of the flight conditions while $\mathrm{X}_{\text {inlet }}$ is also a function of the inlet design itself.

For the sake of simplicity, let us model the combustor as consisting of two separate flow processes: a pressure drop followed by work addition through the combustion of fuel. If we define an intermediate station inside of the combustor (denoted as $3_{\mathrm{a}}$ ) such that station 3a experiences a pressure drop but not an increased temperature, then the work potential at station $3 \mathrm{a}$ is simply equal to the product of $\mathrm{w}_{3}$ times the transfer function of the pressure drop, $\mathrm{X}_{\Delta \mathrm{P} / \mathrm{P}}=\mathrm{w}_{3 \mathrm{a}} / \mathrm{w}_{3}$. The work potential emerging at station 4 is equal to the work potential at station 3a plus the work potential added through the combustion of fuel, $\mathrm{w}_{\text {fuel }}$.

$$
w_{4}=w_{1} \frac{w_{3}}{w_{1}} \frac{w_{3 a}}{w_{3}}+w_{\text {fuel }}=w_{1} X_{\text {inlet }} X_{\Delta P / P}+w_{\text {fuel }}
$$

Note that $\mathrm{w}_{\text {fuel }}$ is a function of the fuel properties and combustion conditions inside the combustor. In general, it is not the same as fuel exergy or fuel heating value. Rather, it is the usable work potential added due to heat release in the combustor. In the present context, useable work potential is given by the gas specific power, sometimes referred to as gas horsepower, at station 4 minus the gas specific power at station 3 (see Ref. 3 for a detailed definition of gas specific power). The combustion conditions are in turn a function of the ram compression work, and therefore, $\mathrm{w}_{\text {fuel }}=\mathrm{f}\left(\mathrm{w}_{1}\right)$, as we will see later in this discussion.

Continuing with the previous discussion, the work potential at station 4 then passes through the nozzle to emerge at station 9. Hopefully, the great majority of the useable work potential available at station 4 has been transformed into kinetic energy of the exiting flow at station 9. The nozzle is not perfectly efficient and therefore $\mathrm{w}_{9}$ is the product of the work transfer function of the nozzle and $\mathrm{w}_{4}$ :

$$
w_{9}=\left(w_{1} \frac{w_{3}}{w_{1}} \frac{w_{3 a}}{w_{3}}+w_{\text {fuel }}\right) \frac{w_{9}}{w_{4}}=\left(w_{1} X_{\text {inlet }} X_{\Delta P / P}+w_{\text {fuel }}\right) X_{\text {nozzle }}
$$

and the net cycle work available is given by the gross work minus the ram work of initial compression:

$$
w_{\text {cycle }}=\left(w_{1} \frac{w_{3}}{w_{1}} \frac{w_{3 a}}{w_{3}}+w_{\text {fuel }}\right) \frac{w_{9}}{w_{4}}-w_{1}
$$

re-arranging yields the following expression for net cycle work:

$$
w_{\text {cycle }}=w_{\text {fuel }}\left[\frac{w_{9}}{w_{4}}\right]-w_{1}\left[1-\frac{w_{3}}{w_{1}} \frac{w_{3 a}}{w_{3}} \frac{w_{9}}{w_{4}}\right]=w_{\text {fuel }} X_{\text {nozzle }}-w_{1} X_{\text {inlet }} X_{\Delta P / P} X_{\text {nozzle }}
$$

Referring back to Eq. (1), the cycle transfer efficiency for a ramjet can therefore be expressed as:

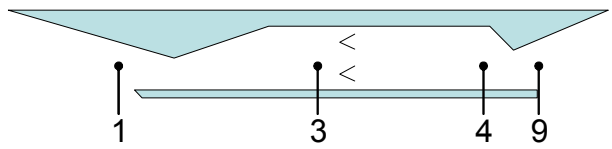

Fig. 1: Typical Ramjet Engine Component and Flow Station Arrangement. 


$$
\eta_{C T}=\frac{w_{\text {cycle }}}{w_{\text {fuel }}}=\underbrace{\left[\frac{w_{9}}{w_{4}}\right]}_{\text {Gross Work Transfer }}-\frac{w_{1}}{w_{\text {fuel }}} \underbrace{\left[1-\frac{w_{3}}{w_{1}} \frac{w_{3 a}}{w_{3}} \frac{w_{9}}{w_{4}}\right]}_{\text {Ram Work Loss Function }}=X_{\text {nozzle }}-\frac{w_{1}}{w_{\text {fuel }}} X_{\text {inlet }} X_{\Delta P / P} X_{\text {nozzle }}
$$

Note that the terminology "effective" cycle efficiency is used here because $\mathrm{w}_{\text {fuel }}$ is not the same as LHV, so this expression is not the same thing as thermal efficiency as we are accustomed to thinking of it. Rather, the product of cycle transfer and cycle availability efficiency is equivalent to the standard definition of thermal efficiency.

The form of Eq. (6) suggests that the transfer of available energy into cycle output in a ramjet cycle has two principal work transfer paths inherent to it: a feedforward path and a feedback path. The term labeled "gross work transfer function" governs how much of the useable fuel work potential, $\mathrm{w}_{\text {fuel }}$, is converted into gross cycle work (i.e., it is the feedforward path taking fuel work potential into gross cycle work). It governs how effectively the work potential available at station 4 is converted into useful cycle work.

The feedback path involves two terms. First the term $\mathrm{w}_{1} / \mathrm{w}_{\text {fuel }}$ is a non-dimensional "gain" parameter quantifying the ram work/net cycle work split as a fraction of fuel work potential input into the system. This parameter is a function of combustion conditions (and therefore flight speed) and fuel properties. The term labeled "ram work loss function" governs how much of the ram work that is fed back into the cycle is lost. In effect, the ram work loss function quantifies the "efficiency" of the ram work transfer path in terms of how much gross cycle work is required to produce a unit of ram compression work in the ramjet cycle.

The equation for net cycle work (Eq. 5) suggests that four parameters drive the total work output in a ramjet machine: the previous three parameters, and specific work potential added to this cycle through the fuel. It is important to note that the preceding conclusions were arrived at by assuming nothing more than the basic arrangement and connectivity of components in a ramjet engine. Absolutely nothing about the component performance, cycle efficiency, propulsive efficiency, air properties, or fuel properties has been assumed at this point. This is a strength of the work transfer analysis method: key groupings of efficiency parameters are a fallout of the analysis.

In view of these results, it is evident that the schematic shown in Fig. 1 is not a convenient representation of work transfer through a ramjet engine. Fig. 1 focuses on the flow of air through the system, not the flow of work potential. Moreover, the ram work transfer loop is not even depicted on the ramjet engine schematic diagram, though it is certainly a real and very important path of work transfer. From a work transfer perspective, the schematic should start with fuel work potential and end with net thrust output. Fig. 2 shows a modified ramjet engine schematic that more accurately reflects the work potential point of view. Note that there is now a loop to capture the ram work transfer and the schematic now begins and ends with work transfer into and out of the propulsion system.

Recall that $\mathrm{w}_{1}$ is the kinetic energy of the incoming flow. The available energy input into the machine, $\mathrm{w}_{\text {fuel }}, \mathrm{is}$ the useable work potential. In other words, $\mathrm{w}_{\text {fuel }}$ is that portion of the fuel enthalpy with the potential to be converted into exhaust kinetic energy. The ratio $\mathrm{w}_{1} / \mathrm{w}_{\text {fuel }}$ is in turn a function of flight speed and fuel heating value. The Eq. (6) clearly shows that as the ratio $\mathrm{w}_{1} / \mathrm{w}_{\text {fuel }}$ increases, it becomes more important for the ram work loss function to decrease. Further, it is clear that the impact of component work transfer becomes increasingly important as the total ram work increases. In general, the component with the lowest work transfer will have the highest sensitivity to total machine output and efficiency. Finally, note that the nozzle work transfer appears in both the ram work path and the feedforward transfer path, and is therefore doubly important. All of these results are congruent with our intuitive expectations for the behavior of ramjet performance as a function of operating condition, fuel heating value, and component performance. However, the above expression of ramjet performance is more

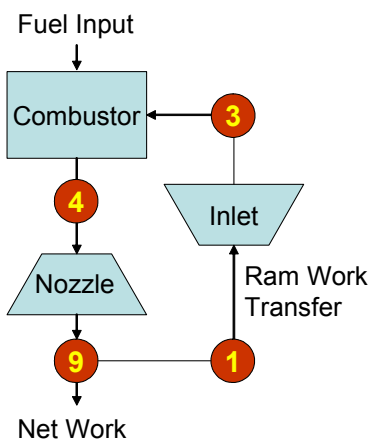

Fig. 2: Ramjet Engine Schematic Viewed from a Work Transfer Point of View. 


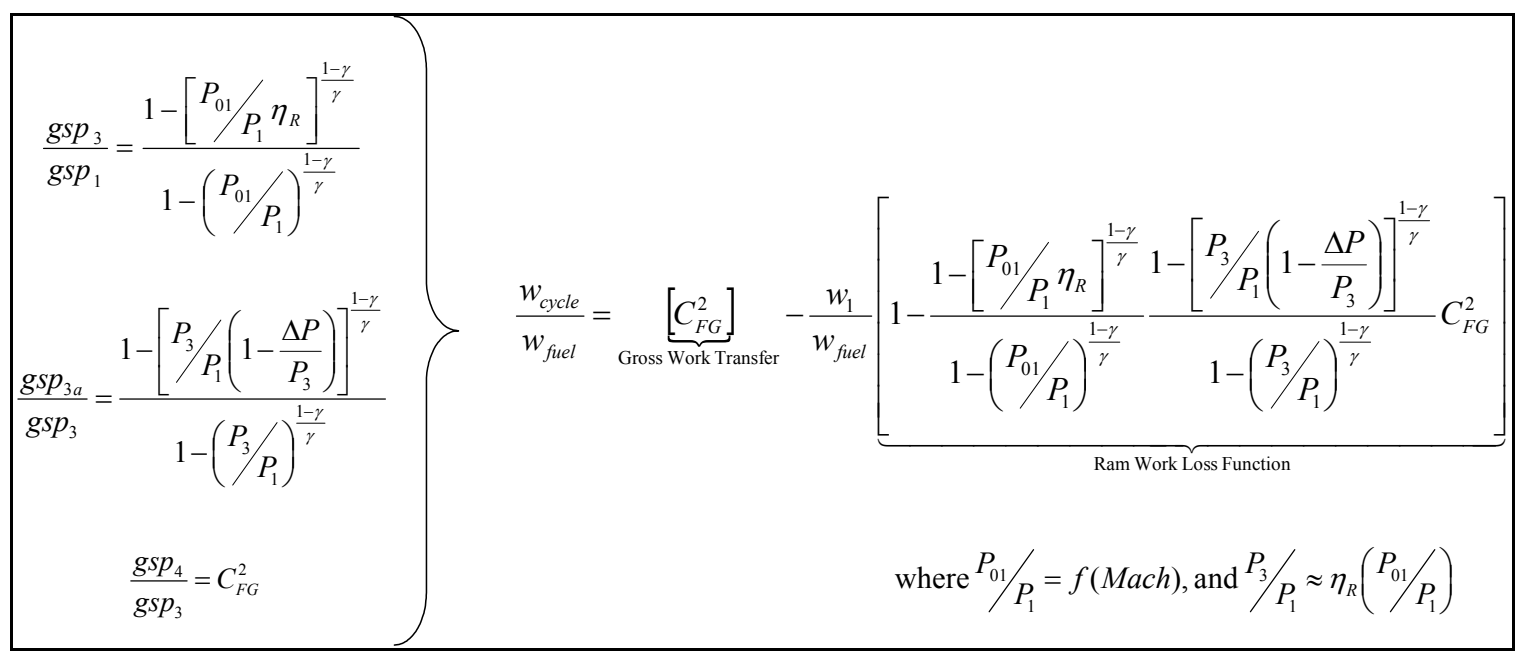

Fig. 3: Ramjet Work Transfer as a function of Classic Component Efficiency Parameters.

compact, concise, and intuitively revealing than the more commonly used performance representations.

The cycle work transfer representation for the ramjet engine can be expressed in terms of classic component efficiencies by using the results derived in Ref. 4, as shown in Fig. 3. It is evident that the nozzle thrust coefficient has a large impact on total transfer of fuel work potential into kinetic energy of the ramjet propulsive stream. Note also that the loss in work transfer is a function of the dimensionless ram work passing through the ram work feedback loop.

If one recognizes that the non-dimensional ram work feedback is a function of flight Mach number, as is the stagnation pressure ratio, then the equation assembled in Fig. 3 can be further re-arranged such that it can be expressed as a function of Mach number:

$\frac{w_{\text {cycle }}}{w_{\text {fuel }}}=\underbrace{\left[C_{F G}^{2}\right]}_{\text {Gross Work Transfer }}-\frac{\frac{M^{2} \gamma R T_{1}}{2}}{w_{\text {fuel }}}\left\{1-\left[\frac{1-\left[1+\frac{\gamma-1}{2} M^{2}\right]^{-1} \eta_{R}^{\frac{1-\gamma}{\gamma}}}{1-\left[1+\frac{\gamma-1}{2} M^{2}\right]^{-1}}\right] \frac{\left.\left.1-\left[1+\frac{\gamma-1}{2} M^{2}\right]^{-1} \eta_{R}^{\frac{1-\gamma}{\gamma}}\left(1-\frac{\Delta P}{P_{2}}\right)^{\frac{1-\gamma}{\gamma}}\right]\left[C_{F G}^{2}\right]\right\}}{1-\left[1+\frac{\gamma-1}{2} M^{2}\right]^{-1} \eta_{R}^{\frac{1-\gamma}{\gamma}}}\right.$

This equation is plotted as a function of Mach number in Fig. 4 for typical values of the remaining parameters. This plot shows how ramjet cycle transfer efficiency changes with Mach number (assuming that component performance is constant with Mach). Note that cycle work transfer decreases as Mach number is increased even when all component efficiencies remain constant. Clearly, efficiency and work transfer are not the same.

\section{B. Ramjet Cycle Availability Efficiency}

Next, let us examine cycle availability efficiency for a ramjet. It should be evident that the cycle availability efficiency must somehow be related to the ram work transfer and ram work loss function derived in the previous section. To see this, note that the cycle pressure ratio expression for cycle availability efficiency (derived in Ref. 1). If we neglect the change in ratio of specific heats between stations $3 a$ and 4 , then cycle availability can be approximated in terms of the work potential at station 3a:

$$
\eta_{C A} \equiv \frac{\text { work potential added due to combustion }}{\text { heat of combustion }}=1-\left(P_{\text {comb }} / P_{a m b}\right)^{\frac{1-\gamma}{\gamma}} \approx \frac{w_{3 a}}{h_{3 a}}
$$

The work potential at station $3 \mathrm{a}$ can be expressed in terms of the work potential at station 1 and the transfer function through the inlet and combustor pressure drop. The enthalpy station $3 \mathrm{a}$ is the same as that at station 1 , which is the same as the initial ram work input, $\mathrm{w}_{1}$.

$$
\eta_{C A} \approx \frac{w_{3 a}}{h_{3 a}}=\left(w_{1} \frac{w_{3}}{w_{1}} \frac{w_{3 a}}{w_{3}}\right) 1 / w_{1}=\frac{w_{3}}{w_{1}} \frac{w_{3 a}}{w_{3}}
$$




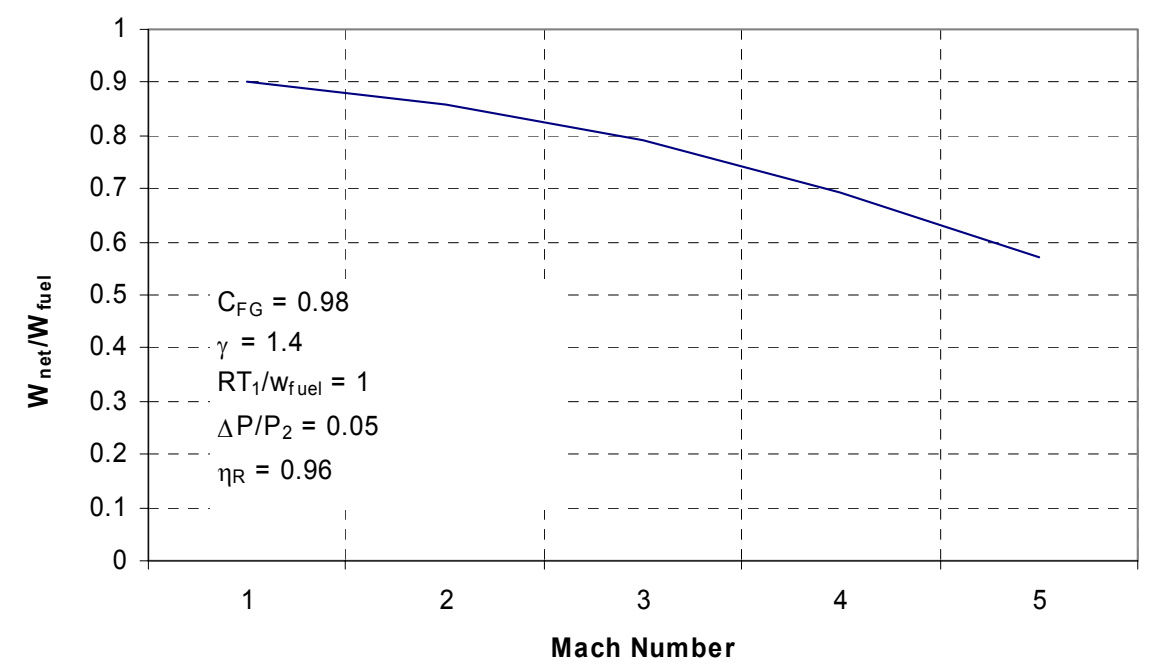

Fig. 4: Ramjet Cycle Work Transfer as a Function of Flight Mach Number.

Combining these results with Eq. (6), we see that cycle work output can be expressed in terms of the cycle availability efficiency and fuel heating value:

$$
w_{\text {cycle }}=L H V \eta_{C A}\left\{\left[\frac{w_{4}}{w_{3}}\right]-w_{1}\left[1-\frac{w_{3}}{w_{1}} \frac{w_{3 a}}{w_{3}} \frac{w_{4}}{w_{3 a}}\right]\right\}=L H V \underbrace{\frac{w_{3}}{w_{1}} \frac{w_{3 a}}{w_{3}}}_{\eta_{C A}}\left\{\left[\frac{w_{4}}{w_{3 a}}\right]-w_{1}\left[1-\frac{w_{3}}{w_{1}} \frac{w_{3 a}}{w_{3}} \frac{w_{4}}{w_{3 a}}\right]\right\}
$$

This expression clearly demonstrates the role of ram work feedback in the ramjet engine cycle. Although the work transfer loop itself contributes only loss to the cycle transfer efficiency, the cycle availability efficiency is directly proportional to the ram work per unit mass, $\mathrm{w}_{1}$, while components in the compression path (inlet work transfer function and combustor pressure drop work transfer function) act to decrease the transfer of fuel energy into available energy.

\section{Ramjet Propulsive Efficiency Expressed as Work Transfer}

It is noted in Ref. 1 that propulsive efficiency is intimately related to both kinetic energy of the incoming flow $\left(\mathrm{w}_{1}\right)$ and the change in kinetic energy of the propulsive flow in passing through the machine. For a single flow machine, propulsive efficiency can be expressed as:

$$
\eta_{P}=\frac{2}{1+u_{e} / u_{0}}=\frac{2}{1+\sqrt{w_{K E} / w_{\text {ram }}}}
$$

Substituting Eq. 10 into this expression and noting that $\mathrm{w}_{\mathrm{cycle}}=\mathrm{w}_{\mathrm{KE}}$ (since transfer efficiency is presumed to be one for a single flow ramjet) yields:

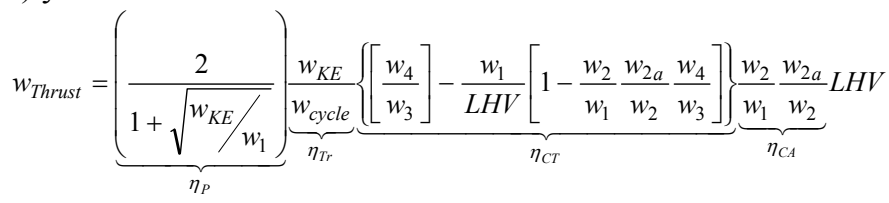

The transfer efficiency of a ramjet engine is 1.0 since the work potential is not transferred into a secondary stream as in the case of a turbofan engine. This equation shows that the efficiency of converting fuel work potential into net cycle output in a ramjet engine is given by the term in the curly braces on the right side. The efficiency of transferring this work potential into thrust work is given by the term in the parentheses. Note that this expression is a nonlinear function of net work output.

\section{Work Transfer in Turbojet Engines}

A similar work transfer analysis to that used for the ramjet can be carried out for the turbojet engine, with the development being a simple extension of the foregoing development for the ramjet engine wherein an additional work transfer loop is added by virtue of the compressor-shaft-turbine added to the flowpath. Using the station 


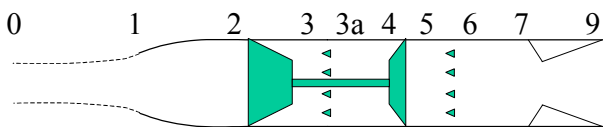

Fig. 5: Turbojet Engine Designations.

designations shown in Fig. 5, an equation for the cycle specific work and cycle transfer efficiency can be derived by inspection. To see how this is done, let us step through the process starting with the work potential present at station $1: \mathrm{w}_{1}=$ (inlet kinetic energy of flow) $=($ ram compression work). The work potential at station 2 is given by:

$$
w_{2}=w_{1} \frac{w_{2}}{w_{1}} .
$$

The work potential going into the compressor component is given by:

$$
w_{\text {in, compressor }}=w_{1} \frac{w_{2}}{w_{1}}+w_{s h}
$$

and the total work potential out of the compressor is, by definition:

$$
w_{3}=\left(w_{1} \frac{w_{2}}{w_{1}}+w_{s h}\right) \frac{w_{3}}{w_{2}} .
$$

Continuing in this same vein, the total work potential entering the combustor is given by:

$$
w_{\text {in ,combustor }}=\left(w_{1} \frac{w_{2}}{w_{1}}+w_{s h}\right) \frac{w_{3}}{w_{2}} \frac{w_{3 a}}{w_{3}}+w_{\text {fuel }}
$$

and therefore the total work potential into the turbine is given by:

$$
w_{\text {int turbine }}=\left[\left(w_{1} \frac{w_{2}}{w_{1}}+w_{s h}\right) \frac{w_{3}}{w_{2}} \frac{w_{3 a}}{w_{3}}+w_{\text {fuel }}\right] \frac{w_{5}}{w_{4}} .
$$

The total work potential at station 6 must therefore be given by:

$$
w_{6}=\left\{\left[\left(w_{1} \frac{w_{2}}{w_{1}}+w_{s h}\right) \frac{w_{3}}{w_{2}} \frac{w_{3 a}}{w_{3}}+w_{\text {ftel }}\right] \frac{w_{5}}{w_{4}}-w_{s h}\right\} \frac{w_{6}}{w_{5}}
$$

where $\mathrm{w}_{\mathrm{sh}}$ is the shaft work transfer between the turbine and compressor. The total work potential available at station 9 is:

$$
w_{9}=\left\{\left[\left(w_{1} \frac{w_{2}}{w_{1}}+w_{s h}\right) \frac{w_{3}}{w_{2}} \frac{w_{3 a}}{w_{3}}+w_{\text {fuel }}\right] \frac{w_{5}}{w_{4}}-w_{\text {sh }}\right\} \frac{w_{6}}{w_{5}} \frac{w_{9}}{w_{7}} .
$$

Finally, the net work potential output from the cycle is given by $w_{9}$ minus the work of ram compression:

Collecting terms yields:

$$
w_{\text {cycle }}=\left\{\left[\left(w_{1} \frac{w_{2}}{w_{1}}+w_{s h}\right) \frac{w_{3}}{w_{2}} \frac{w_{3 a}}{w_{3}}+w_{\text {fuel }}\right] \frac{w_{5}}{w_{4}}-w_{s h}\right\} \frac{w_{6}}{w_{5}} \frac{w_{9}}{w_{7}}-w_{1} .
$$

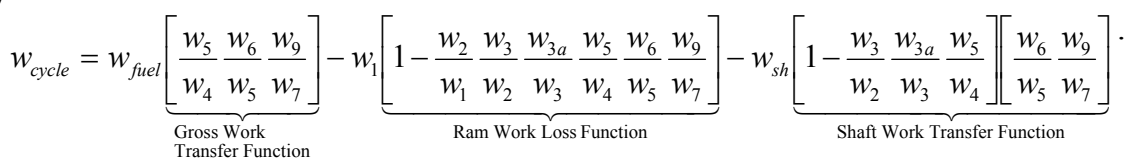

Using a similar approach to that employed for the ramjet, one can obtain an expression for the cycle work transfer efficiency of the turbojet system:

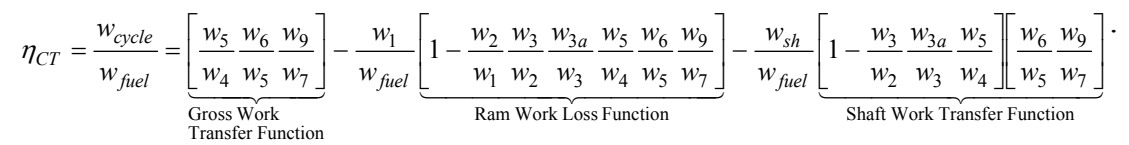

Note that the gross work feedworward term remains largely unchanged from the ramjet case, though an extra component work transfer term has been added to account for the presence of the turbine in the feedforward path. Likewise, the ram work loss function is basically unchanged, though more work transfer terms are included to account for the various components in the ram work feedback loop path. One new term appears in this equation to account for the new work transfer path from the turbine into the compressor. Note that this term contains several parts: the total shaft work, the shaft work loss function, and a feedforward term. The loss function contains only those component transfer functions present in the shaft work feedback path. The feedforward term contains only those components in the path from the turbine to the thrust nozzle. 


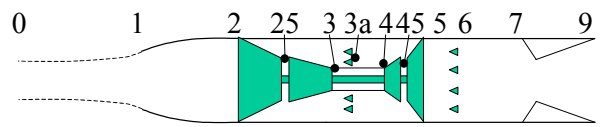

Fig. 6: Dual-Spool Turbojet Engine Station Designations.

For an afterburning turbojet engine (where it has again been assumed that the combustion process consists of a pressure drop followed by heat addition), one additional term appears in the equation:

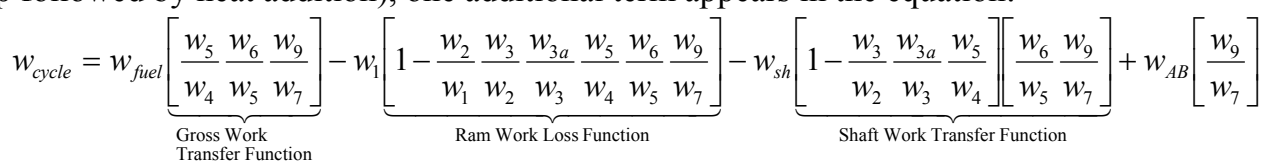

where $\mathrm{w}_{\mathrm{AB}}$ is the work potential associated with the addition of fuel in the afterburner. An expression for overall cycle work transfer efficiency must now include the total work potential input into the system:

$$
\frac{w_{\text {cycle }}}{w_{\text {tot }}}=\frac{w_{\text {fuel }}}{w_{\text {tot }}} \underbrace{\left[\frac{w_{5}}{w_{4}} \frac{w_{6}}{w_{5}} \frac{w_{9}}{w_{7}}\right]}_{\text {Gross Combustor Work }}+\frac{w_{A B}}{w_{\text {tot }}} \underbrace{\left[\frac{w_{9}}{w_{7}}\right]}_{\text {AB Gross Work Xfr Function }}-\frac{w_{1}}{w_{\text {tot }}} \underbrace{\left[1-\frac{w_{2}}{w_{1}} \frac{w_{3}}{w_{2}} \frac{w_{3 a}}{w_{3}} \frac{w_{5}}{w_{4}} \frac{w_{6}}{w_{5}} \frac{w_{9}}{w_{7}}\right]}_{\text {Ram Work Loss Function }}-\frac{w_{s h}}{w_{\text {tot }}} \underbrace{\left[1-\frac{w_{3}}{w_{2}} \frac{w_{3 a}}{w_{3}} \frac{w_{5}}{w_{4}}\right]\left[\frac{w_{6}}{w_{5}} \frac{w_{9}}{w_{7}}\right]}_{\text {Shaft Work Transfer Function }}
$$

where $\mathrm{w}_{\text {tot }}=\mathrm{w}_{\text {fuel }}+\mathrm{w}_{\mathrm{AB}}$.

Extending this analysis further to a dual-spool turbojet engine, using engine station designations given in Fig. 6, the work transfer is given by:

$$
\begin{aligned}
& w_{\text {cycle }}=w_{\text {fuel }} \underbrace{\left[\frac{w_{45}}{w_{4}} \frac{w_{5}}{w_{45}} \frac{w_{6}}{w_{5}} \frac{w_{9}}{w_{7}}\right]}_{\text {GrossWorkTransfer }}-w_{1} \underbrace{\left[1-\frac{w_{2}}{w_{1}} \frac{w_{25}}{w_{2}} \frac{w_{3}}{w_{25}} \frac{w_{3 a}}{w_{3}} \frac{w_{45}}{w_{4}} \frac{w_{5}}{w_{45}} \frac{w_{6}}{w_{5}} \frac{w_{9}}{w_{7}}\right]}_{\text {RamWorkLossFinction }}-w_{l o}^{\left[\frac{w_{25}}{w_{2}} \frac{w_{3}}{w_{25}} \frac{w_{3 a}}{w_{3}} \frac{w_{45}}{w_{4}} \frac{w_{5}}{w_{45}}-1\right]}\left[\frac{w_{6}}{w_{5}} \frac{w_{9}}{w_{7}}\right]-\ldots \\
& w_{h i} \underbrace{\left[\frac{w_{3}}{w_{25}} \frac{w_{3 a}}{w_{3}} \frac{w_{45}}{w_{4}}-1\right]}_{\text {Hi-SpoolLossFunction }}\left[\frac{w_{5}}{w_{45}} \frac{w_{6}}{w_{5}} \frac{w_{9}}{w_{7}}\right]
\end{aligned}
$$

One can begin to notice a pattern at this point with respect to the derivation of system work transfer functions:

- $\quad$ First, there will be one work feedforward term for each source of work potential injected into the system. This feedforward term governs how much of the useable work potential injected into the system is ultimately manifested as useable gross thrust work. Furthermore, the component transfer functions contained in this feedforward term are those in the transfer path from the point of work injection to the point of system work output.

- Second, there is a single work loss function for each work feedback loop. The terms in the loss function are those components contained in the feedback loop itself. The presence of a work feedback loop may also give rise to a feedforward term, and the component transfer functions in that term consist of those in the transfer path from the feedback loop to the system output.

- At no time during this development was it necessary to invoke the laws of thermodynamics or the laws of motion, with the exception of the second law of thermodynamics. The second law is implicitly used in the form: $\mathrm{w}_{\text {out }}=\left(\Sigma \mathrm{W}_{\text {in }}\right) \mathrm{X}_{\text {component }}$, or in other words, the total work potential output from a component must be equal to the sum of the input work potential multiplied by some work transfer function which must always be less than or equal to 1.0 .

These are general rules which hold for all work transfer systems. Many additional sources of loss could be included in the basic model outlined above, but the form of the equations would remain the same. The concept is illustrated diagrammatically in Fig. 7 for the dual-spool turbojet example mentioned previously. The basic dualspool machine can be decomposed into a single feedforward path and three work feedback paths. Each feedback path in turn consists of a loss function (constructed by traversing around the loop) and a feedforward function (created by traversing from the nearest edge of the loop to the system output). In this way, a work transfer representation can readily be derived for most propulsion systems of interest by inspection of the component arrangement.

\section{Work Transfer in Turbofan Engines}

The previous discussion and observations regarding the decomposition of a complex work transfer system into simpler work feedforward and feedback systems will greatly facilitate the derivation of analogous expressions for separate and mixed flow turbofan engines. Let us start by considering the dual-spool separate flow turbofan having 

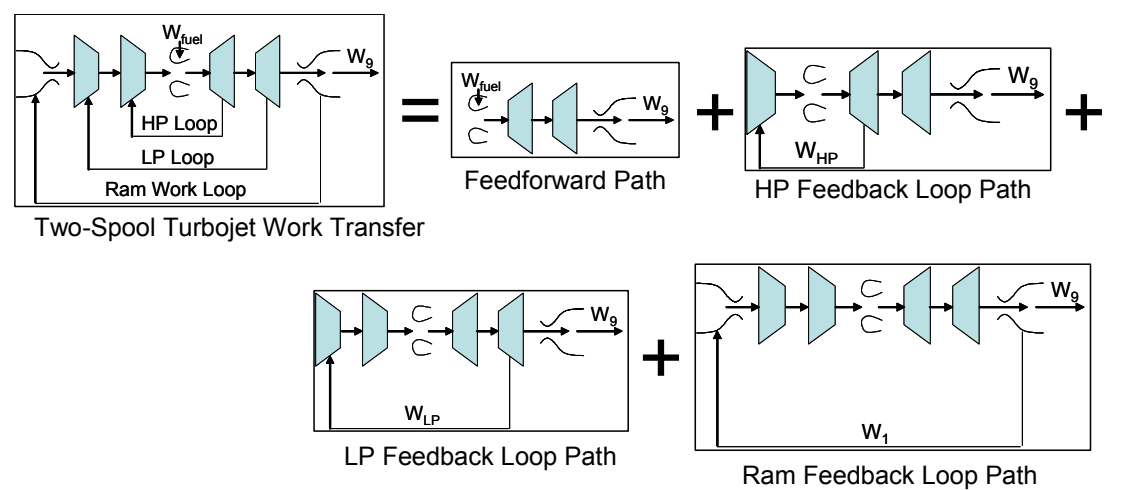

Fig. 7: Dual-Spool Turbojet Engine Decomposed into Basic Work Transfer Paths.

the arrangement shown in Fig. 8. To do so, presume that the two-spool turbojet considered previously is the core of a separate flow turbofan engine, where the fan is appended to the front of the LP spool and the fan hub + LP compressor now becomes a booster modeled as a single unit.

The equivalent work transfer representation of this cycle is shown in Fig. 9. For the sake of analytical convenience, the engine is treated as having two separate flow streams, each undergoing its own work transfer process. Work transfer in the core stream is almost identical to the dual-spool turbojet case, though with a portion of the LP shaft work being transferred into the bypass stream. The work transfer process undergone by the bypass stream looks very similar to that undergone by the ramjet, though with the important difference that the work potential transferred into the system is not a function of inlet kinetic energy (ram work transfer) the way it is for the ramjet.

Since the separate flow turbofan has two mechanisms for work output (fan thrust and core nozzle thrust), the work transfer analysis used previously for the turbojet must be modified accordingly. In this case, the DSTJ net work output equation must have an additional term to account for the extraction of shaft work used to drive the fan:

$$
w_{\text {net,core }}=(\text { D.S.T.J. })-w_{\text {fan }}\left[\frac{w_{6}}{w_{5}} \frac{w_{9}}{w_{7}}\right]
$$

Since there is also a fan stream into which the fan shaft work is placed, there must be one additional work transfer equation for the fan stream:

$$
w_{\text {net, fan }}=\left(w_{12} \frac{w_{12}}{w_{11}}+w_{\text {fan }}\right)\left[\frac{w_{16}}{w_{12}} \frac{w_{19}}{w_{16}}\right]-w_{12}=w_{\text {fan }}\left[\frac{w_{16}}{w_{12}} \frac{w_{19}}{w_{16}}\right]-w_{12}\left(1-\left[\frac{w_{12}}{w_{11}} \frac{w_{16}}{w_{12}} \frac{w_{19}}{w_{16}}\right]\right)
$$

where this model assumes the only losses in the fan stream are in the inlet, fan, and fan nozzle. This equation can be combined with the core work expression to yield the total net work output:

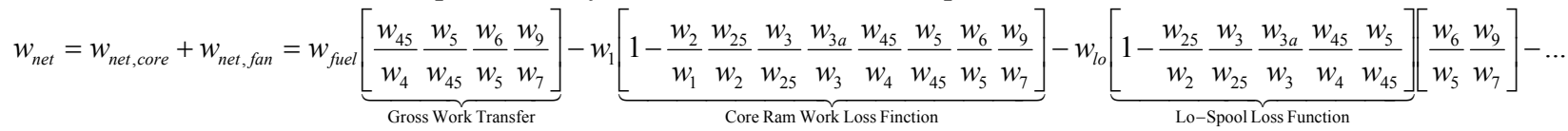

$$
\begin{aligned}
& w_{h i} \underbrace{\left[1-\frac{w_{3}}{w_{25}} \frac{w_{3 a}}{w_{3}} \frac{w_{45}}{w_{4}}\right]}_{\text {Hi-Spool Loss Function }}\left[\frac{w_{5}}{w_{45}} \frac{w_{6}}{w_{5}} \frac{w_{9}}{w_{7}}\right]+w_{\text {fan }}^{\left[\frac{w_{16}}{w_{12}} \frac{w_{19}}{w_{16}}-\frac{w_{6}}{w_{5}} \frac{w_{9}}{w_{7}}\right]}-\underbrace{w_{12}}_{\text {Fan Work Transfer Function }} \underbrace{\left[1-\frac{w_{12}}{w_{11}} \frac{w_{16}}{w_{12}} \frac{w_{19}}{w_{16}}\right]}_{\text {Fan Ram Wk Loss Fn }}
\end{aligned}
$$

Comparing this equation with its two-spool turbojet counterpart, it is evident that the two additional terms at the end of the expression can either increase or decrease the net work output, depending on the relative magnitudes of the contributing component transfer functions. Specifically, the fan work transfer function can add or detract from

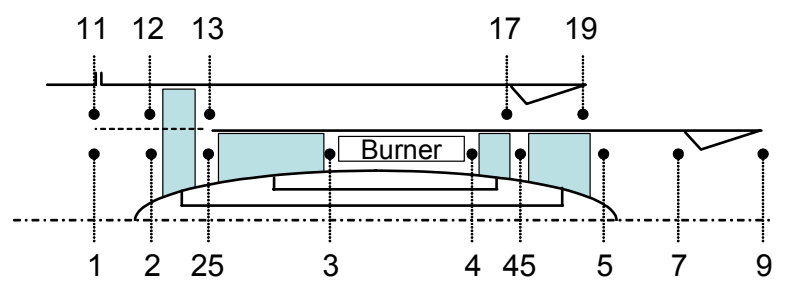

Fig. 8: Dual-Spool Separate Flow Turbofan Station Designations (After SAE Aerospace Recommended Practice 755). 

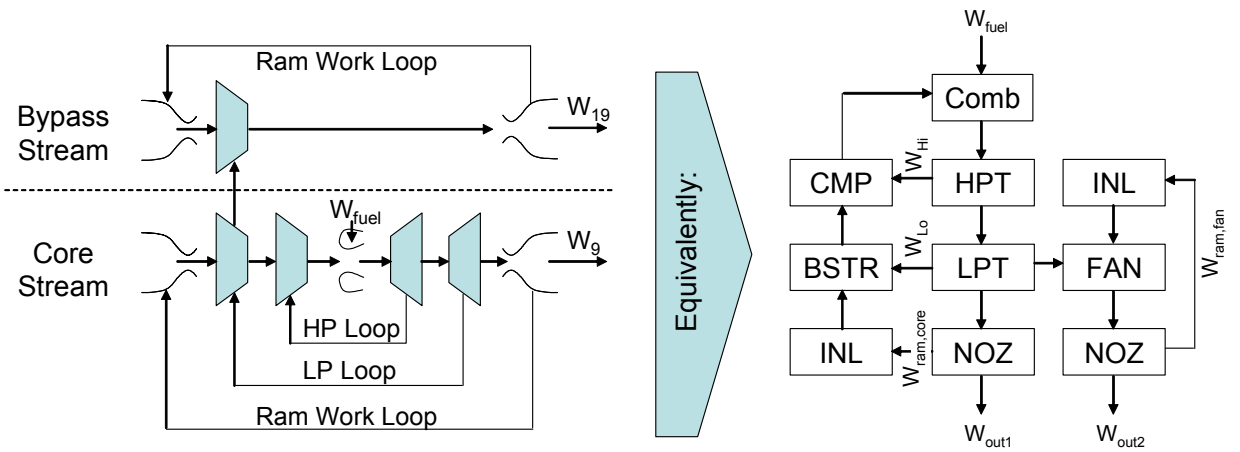

Fig. 9: Work Transfer Representation of a Dual-Spool Turbofan Engine.

total cycle work output, depending on the relative magnitudes of fan work transfer versus core nozzle work transfer. This equation suggest that the product of fan and fan nozzle transfer efficiency should be higher than the product of LPT and core nozzle transfer efficiency if the addition of the fan stream is to be a favorable trade over the plain turbojet. The fan ram work path is always negative in sign, but the work transfer across the total loss can usually be made very small because the product of fan inlet, fan, and fan nozzle work transfer is very close to 1.0.

Focus for a moment on the portion of the schematic that represents the transfer of fan shaft work into production of fan stream kinetic energy (denoted as $\mathrm{w}_{\text {fan }}$ ). The transfer of work potential from gas generator shaft work into fan propulsive stream kinetic energy was previously labeled the transfer efficiency. Referring back to the block diagram algebra, it should be apparent that the fan work transfer path is a simple feedback loop that can be represented as:

$$
w_{K E, \text { fan }}=w_{\text {fan }} X_{\text {fan }} X_{\text {fannozzle }}-\left(1-X_{\text {inlet }} X_{\text {fan }} X_{\text {fannozzle }}\right) w_{12}
$$

where: $\quad X_{i}=$ work transfer function for component ' $i$ '

$\mathrm{w}_{\mathrm{fan}}=$ shaft work into the fan (bypass stream only)

$\mathrm{w}_{12}=$ ram work of incoming fan stream (bypass stream only)

Note that the more convenient notation of component work transfer as " $\mathrm{X}_{\mathrm{i}}$ " is henceforth being used in order to keep the equations as compact and comprehensible as possible. The transfer efficiency is therefore given by:

$$
\eta_{T r, \text { fan }}=\frac{w_{K E, \text { fan }}}{w_{\text {fan }}}=X_{\text {fan }} X_{\text {fannozzle }}-\left(1-X_{\text {inlet }} X_{\text {fan }} X_{\text {fannozzle }}\right) \frac{w_{12}}{w_{\text {fan }}}
$$

This equation shows that the transfer efficiency of the fan system is given by the feedforward function, the loss function, and the ratio of fan ram work to fan shaft work (where lower case $\mathrm{w}$ is again presumed to mean work per pound of flow). It is safe to presume that the work transfer functions for the fan and fan nozzle have a good deal more impact on transfer efficiency than does the inlet work transfer. Also, it is clear from this expression that transfer efficiency is highly dependent on the non-dimensional ram work transfer.

\section{A. Typical Design Point Work Transfer (Separate Flow Turbofan)}

One can obtain a feel for the magnitudes of the terms in Eq. (28) for a typical high bypass turbofan engine by assuming typical component efficiencies and using the equations derived in Ref. 4. Let us assume for the sake of argument a machine having an overall pressure ratio of 45 , a fan pressure ratio of 1.45 , a compressor pressure ratio of 22, a bypass ratio of 9:1, and a design point turbine inlet temperature of 3,300 R (1833 K). Further assume the design point component efficiencies as shown in Table 1. All cooling flow circuits are ignored in the interest of simplifying the analysis, though they could be included in the analysis if desired.

\begin{tabular}{|c|c|c|c|}
\hline Parameter & Design Point Value & Work Transfer & Eq. in Ref. 4 \\
\hline Fan Adiabatic Efficiency & 0.92 & 0.9281 & $(37)$ \\
\hline Booster Adiabatic Efficiency & 0.90 & 0.9566 & (36) \\
\hline Compressor Adiabatic Efficiency & 0.87 & 0.9601 & (36) \\
\hline Combustor $\Delta \mathrm{P} / \mathrm{P}$ & $5 \%$ & 0.9925 & (22) \\
\hline HPT Adiabatic Efficiency & 0.92 & 0.9634 & (41) \\
\hline LPT Adiabatic Efficiency & 0.94 & 0.7710 & (41) \\
\hline Core Nozzle Thrust Coefficient & 0.99 & 0.9801 & (5) \\
\hline Fan Nozzle Thrust Coefficient & 0.99 & 0.9801 & (5) \\
\hline
\end{tabular}

Table 1: Typical Design Point Component Parameters for a Commercial Turbofan Engine. 
Let us substitute these values into the work transfer function derived in Eq. (28), examining each work transfer path individually. Beginning with the feedforward work transfer:

$$
w_{\text {fuel }}\left[\frac{w_{45}}{w_{4}} \frac{w_{5}}{w_{45}} \frac{w_{6}}{w_{5}} \frac{w_{9}}{w_{7}}\right]=w_{\text {fuel }}[(0.9634)(0.7710)(1.0)(0.9801)]=0.7280 w_{\text {fuel }}
$$

The feedforward work transfer path is $73 \%$ efficient. Next, considering the core ram compression work transfer:

$$
w_{1}\left[1-\frac{w_{2}}{w_{1}} \frac{w_{25}}{w_{2}} \frac{w_{3}}{w_{25}} \frac{w_{3 a}}{w_{3}} \frac{w_{45}}{w_{4}} \frac{w_{5}}{w_{45}} \frac{w_{6}}{w_{5}} \frac{w_{9}}{w_{7}}\right]=w_{1}[1-(1.0)(0.9566)(0.9601)(0.9925)(0.9634)(0.7710)(1.0)(0.9801)]=0.3364 w_{1}
$$

Thus, $34 \%$ of the core ram compression work is lost in the ram work transfer loop (or in other words, the core ram compression work transfer is $66 \%$ efficient. Next, the low spool work transfer is given by:

$$
w_{l o}\left[1-\frac{w_{25}}{w_{2}} \frac{w_{3}}{w_{25}} \frac{w_{3 a}}{w_{3}} \frac{w_{45}}{w_{4}} \frac{w_{5}}{w_{45}}\right]\left[\frac{w_{6}}{w_{5}} \frac{w_{9}}{w_{7}}\right]=w_{l o}[1-(1.0)(0.9566)(0.9601)(0.9925)(0.9634)(0.7710)][(1.0)(0.9801)]=0.3165 w_{l o}(35)
$$

The low spool work transfer path is roughly $68 \%$ efficient. Considering the high spool transfer path:

$$
w_{h i}\left[1-\frac{w_{3}}{w_{25}} \frac{w_{3 a}}{w_{3}} \frac{w_{45}}{w_{4}}\right]\left[\frac{w_{5}}{w_{45}} \frac{w_{6}}{w_{5}} \frac{w_{9}}{w_{7}}\right]=w_{h i}[1-(1.0)(0.9566)(0.9601)(0.9925)(0.9634)][(0.7710)(1.0)(0.9801)]=0.0921 w_{h i}
$$

Note that the high spool work transfer is quite efficient, with better than $90 \%$ transfer efficiency. Next, let us examine the work transfer in the fan ram compression transfer path:

$$
w_{12}\left[1-\frac{w_{12}}{w_{11}} \frac{w_{16}}{w_{12}}\right]=w_{12}[1-(0.9281)(0.9801)]=0.0904 w_{12}
$$

Not surprisingly, the fan ram work transfer path is highly efficient (91\%). Finally, let us examine the work transfer path between the core stream and the fan stream:

$$
w_{\text {fan }}\left[\frac{w_{16}}{w_{12}} \frac{w_{19}}{w_{16}}-\frac{w_{6}}{w_{5}} \frac{w_{9}}{w_{7}}\right]=w_{\text {fan }}[(0.9281)(0.9801)-(0.1 .0)(0.9801)]=-0.0705 w_{\text {fan }}
$$

This last term is of opposite sign to the previous terms, which implies that $7 \%$ of the shaft work transferred through the LP shaft and into the fan stream is lost, with the remainder being available to offset fan stream ram work losses and contribute to net thrust work output. Collecting the above terms yields a single expression for cycle transfer efficiency in Eq. 39. Similar expressions can be developed for cycle availability efficiency, transfer efficiency, etc.

$$
w_{\text {net }}=0.7280 w_{\text {fuel }}-0.3364 w_{1}-0.3165 w_{l o}-0.0921 w_{h i}-0.0904 w_{12}-0.0705 w_{\text {fan }}
$$

\section{B. Work Transfer in a Mixed Flow Turbofan}

A similar analysis can be applied to a mixed flow turbofan engine. A typical block diagram for a mixed flow turbofan engine is shown in Fig. 10. Note that this diagram contains three nested loops in addition to a feedforward loop. One significant difference, however, is that there is only a single work input and output path, so the definitions for cycle efficiency and work transfer defined previously can be applied directly. An expression for work transfer through this system is given by:

$$
\begin{aligned}
& w_{n e t}=w_{\text {fuel }} \underbrace{X_{E} X_{F} X_{G} X_{H} X_{I}}_{\text {FeedforwardWorkTransfer }}-w_{\text {ram }} \underbrace{\left[1-X_{A} X_{B} X_{C} X_{D} X_{E} X_{F} X_{G} X_{H} X_{I}\right.}_{\text {RamWorkLossFinction }}]-w_{\text {sh }} \underbrace{1-X_{B} X_{C} X_{D} X_{E} X_{F} X_{G}}_{\text {Lo-SpoolLossFunction }}]\left[X_{H} X_{I}\right]+\ldots \\
& -w_{\text {sh }} \underbrace{1-X_{D} X_{E} X_{F}}_{\text {Hi-SpoolLossFunction }}\left[X_{G} X_{H} X_{I}\right]+w_{\text {bypass }} \underbrace{X_{J}-X_{D} X_{E} X_{F} X_{G}}_{\text {FeedforvardGainFunction }} \llbracket X_{H} X_{I}]
\end{aligned}
$$

As before, let us start by considering the transfer efficiency. In the case of the separate flow turbofan engine, transfer efficiency was dominated by the transfer of fan shaft work into a change of kinetic energy in the bypass stream. In the case of the mixed flow turbofan, there is only one propulsive output stream which is not dominated

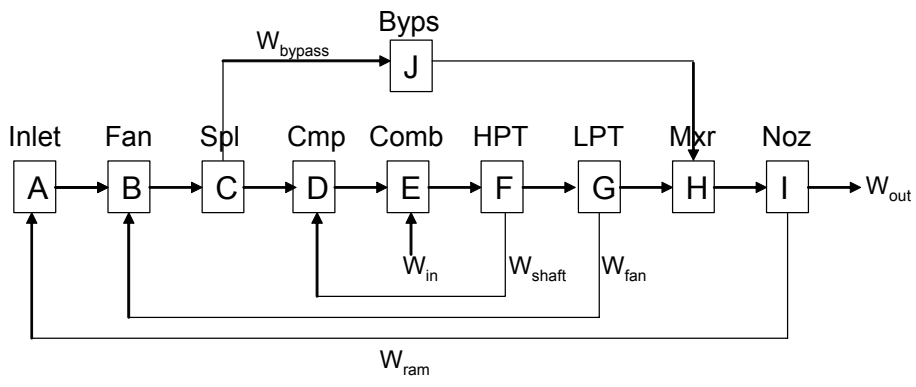

Fig. 10: Block Diagram Representation for a Mixed Flow Turbofan Engine. 
by either the bypass or core streams. Therefore, the entire collection of components A-G as well as J "look" like a gas generator in that their function is to transfer work theoretically available to the cycle into useable work potential into the mixer. If viewed in this way, the component that controls the transformation of cycle net work potential output into a change of propulsive stream kinetic energy is the nozzle. Therefore, the transfer efficiency can be thought of as being given by the nozzle work transfer efficiency:

$$
\eta_{T r}=\frac{w_{K E}}{w_{\text {tailpipe }}}=X_{I}
$$

where $\mathrm{w}_{\mathrm{KE}}$ is the net kinetic energy deposited into the propulsive stream and $\mathrm{w}_{\text {tailpipe }}$ is the work potential entering the nozzle from the tailpipe.

The cycle transfer efficiency is then defined as the transfer of work potential available to the ideal cycle into work potential in the tailpipe. We know from the definitions of cycle transfer efficiency and transfer efficiency that their product is equal to the net work output divided by the fuel work potential input. An expression for this quantity was derived previously. If the quantity representing the transfer efficiency is factored out, the remaining terms represent the cycle transfer efficiency. In this case, if $\mathrm{X}_{\mathrm{H}}$ is factored out of the net work transfer expression, the cycle transfer efficiency is given by:

$$
\begin{aligned}
& \eta_{C T}=\frac{w_{\text {net }}}{w_{\text {fuel }}}=\underbrace{X_{E} X_{F} X_{G} X_{H} X_{I}}_{\text {FeedforvardWorkTransfer }}-\frac{w_{\text {ram }}}{w_{\text {fuel }}} \underbrace{\left.\frac{1}{X_{I}}-X_{A} X_{B} X_{C} X_{D} X_{E} X_{F} X_{G} X_{H}\right]}_{\text {RamWorkLossFinction }}-w_{l o}[\underbrace{1-X_{B} X_{C} X_{D} X_{E} X_{F} X_{G}}_{\text {Lo-SpoolLossFunction }}] X_{H}]+\ldots \\
& -w_{h i} \underbrace{1-X_{D} X_{E} X_{F}}_{H i-\text { SpoolLossFrunction }}]\left[X_{G} X_{H}\right]+w_{\text {bypass }} \underbrace{\left[X_{J}-X_{D} X_{E} X_{F} X_{G}\right]}_{\text {FeedforvardGainFunction }}] X_{H}]
\end{aligned}
$$

Further derivations are possible using the basic ideas outlined herein. These are left for future development.

\section{Conclusion}

The concept of a work transfer function allows all types of thermodynamic systems to be evaluated using work potential as a single universal figure of merit. It does not require assumptions regarding any single component's performance, though one can make assumptions and incorporate them into the analysis if desired. Component work transfer can be related to conventional component efficiencies using a variety of simple formulas. Therefore, the ideas presented herein can easily be integrated into current analysis methods to yield complementary results.

Work transfer analysis reveals important groupings of parameters with nothing more than an assumption regarding the components and their connectivity. Typically, one grouping is obtained per work input stream and work feedback stream. Expressions for cycle transfer efficiency can be derived from inspection of the component block diagram. For constant pressure combustion, the cycle availability efficiency is given by the so-called availability coefficient, which is in turn a function of the work potential of the stream entering the combustor. Therefore, work feedback loops present in turbofan engines act to increase cycle availability efficiency by virtue of increasing the work potential (and pressure) entering the combustor.

Work transfer analysis of ramjet, turbojet, and turbofan engines show some common characteristics, the most prominent of which is the presence of a feedforward work transfer function for each source of work potential introduced into the system and a work loss function for each work feedback loop present in the cycle. The reader will note that work feedback loops have two distinct parts, one being the total work potential transferred back into the system, and the other being a loss transfer term. The total work feedback term (such as $\mathrm{w}_{1}, \mathrm{w}_{\text {shaft }}$, etc.) relates to the total energy transfer (enthalpy rise) of compression. The work feedback loss function has the form $1-\mathrm{X}_{1} \mathrm{X}_{2} \mathrm{X}_{3} \ldots$ and relates to the entropy rise and pressure rise of the compression process. Thus, these two terms embody the first and second laws, respectively. They are sufficient to uniquely identify a thermodynamic state (actually, change from a reference state) since specific enthalpy and entropy constitute two intensive thermodynamic properties.

\section{Acknowledgments}

The author would like to thank NASA Glenn Research Center for their support under contract NAS-3-03115.

\section{References}

\footnotetext{
${ }^{1}$ Roth, B.A., “A Work Transfer Perspective of Propulsion System Performance,” AIAA2004-4079.

${ }^{2}$ Lewis, J.H., "Propulsive Efficiency from an Energy Utilization Standpoint," J. Aircraft, Vol. 13, No. 4, Apr 1976, pp. $299-302$.

${ }^{3}$ Roth, B.A., "Comparison of Thermodynamic Loss Models Suitable for Gas Turbine Propulsion," J. Propulsion and Power, Vol. 17, No. 2, March 2001, pp. 324-332.

${ }^{4}$ Roth, B., "Work Potential Perspective of Engine Component Performance," J. Propulsion and Power, Vol. 18, No. 6, Nov-Dec 2002, pp. 1183-1190.
} 